\title{
Estrogenic Activity of Sanguiin H-6 through Activation of Estrogen Receptor $\alpha$ Coactivator-binding Site
}

\author{
Tuy An Trinh', Eun-Ji Park², Dahae Lee ${ }^{3}$, Ji Hoon Song ${ }^{4}$, Hye Lim Lee ${ }^{5}$, Ki Hyun Kim³ ${ }^{3}$ Younghoon Kim ${ }^{6}$, \\ Kiwon Jung ${ }^{7}$, Ki Sung Kang ${ }^{1, *}$, and Jeong-Eun Yoo $^{2, *}$ \\ ${ }^{1}$ College of Korean Medicine, Gachon University, Seongnam 13120, Korea \\ ${ }^{2}$ Department of Obstetrics and Gynaecology, College of Korean Medicine, Daejeon University, Daejeon 302-869, Korea \\ ${ }^{3}$ School of Pharmacy, Sungkyunkwan University, Suwon 16419, Korea \\ ${ }^{4}$ Department of Medicine, University of Ulsan College of Medicine, Seoul 05505, Korea \\ ${ }^{5}$ Department of Pediatrics, College of Korean Medicine, Daejeon University, Daejeon 302-869, Korea \\ ${ }^{6}$ Pharminogene Inc., Yongin 16827, Korea \\ ${ }^{7}$ Institute of Pharmaceutical Sciences, College of Pharmacy, CHA University, Sungnam 13844, Korea
}

\begin{abstract}
A popular approach for the study of estrogen receptor $\alpha$ inhibition is to investigate the protein-protein interaction between the estrogen receptor (ER) and the coactivator surface. In our study, we investigated phytochemicals from Rubus coreanus that were able to disrupt ER $\alpha$ and coactivator interaction with an ER $\alpha$ antagonist. The E-screen assay and molecular docking analysis were performed to evaluate the effects of the estrogenic activity of $R$. coreanus extract and its constituents on the MCF-7 human breast cancer cell line. At 100 $\mu \mathrm{g} / \mathrm{mL}, R$. coreanus extract significantly stimulated cell proliferation $(574.57 \pm 8.56 \%)$. Sanguiin H6, which was isolated from $R$. coreanus, demonstrated the strongest affinity for the ER $\alpha$ coactivator-binding site in molecular docking analysis, with a binding energy of -250.149 . The initial results of the study indicated that sanguiin H6 contributed to the estrogenic activity of $R$. coreanus through the activation of the ER $\alpha$ coactivator-binding site. Keywords - Rubus coreanus, Sanguiin H-6, Estrogen receptor $\alpha$, Coactivator-binding site, MCF-7 human breast cancer cells.
\end{abstract}

\section{Introduction}

In the last decade, cancer was the leading cause of death in Korea; one in four deaths resulted from the disease. Among Korean women, breast cancer was the second common cancer: cancer statistics from 2013 reported the diagnosis of 17,231 new cases, the occurrence of 2,231 deaths, and 146,416 established cases. ${ }^{1}$ Breast cancer was also predicted to be the most common cancer in Korean women in 2017, with 21,544 estimated new cases and 2,519 estimated deaths. ${ }^{2}$

Owing to the development of science and technology, several new therapies for the treatment of breast cancer have evolved, such as oncoplastic surgery, ${ }^{3}$ bone-directed

\footnotetext{
*Author for correspondence

Ki Sung Kang, College of Korean Medicine, Gachon University, Seongnam 13120, Korea

Tel: +82-31-750-5402; E-mail: kkang@gachon.ac.kr
}

Jeong-Eun Yoo, Department of Obstetrics and Gynaecology, College of Korean Medicine, Daejeon University, Daejeon 302-869, Korea Tel: +82-42-470-9139; E-mail: jeyoo@dju.ac.kr treatments, ${ }^{4}$ and targeted therapy drugs. ${ }^{5}$ In addition to these novel remedial treatments, typical therapies such as tumor excision, adjuvant chemotherapy, or endocrine therapy have also been constantly improved. Estrogen antagonists play an important role in the hormonal manipulation used for treatment of breast cancer. ${ }^{6}$ Tamoxifen has been the most effective drug for breast cancer with estrogen-related tumors for over 30 years. ${ }^{7}$ However, tamoxifen interacts with estrogen receptors other than in the breast cancer cells, which leads to side effects in other tissues. ${ }^{8}$ Screening for new estrogenic antagonists is considered the optimal solution to enhance the efficacy of treatment.

Chemical constituents from herbal plants that exert anticancer effects are expected to provide a potential source of estrogenic antagonists for breast cancer treatment. Numerous published studies have described the anticancer effects of compounds isolated from medicinal plants, such as curcumin from turmeric, thymoquinone from black cumin, 6-shogaol from ginger, diallyl sulfide from garlic, piperine from black pepper, capsaicin from red chili 
pepper, and eugenol from clove. ${ }^{9}$ In spite of these compounds, few researchers have compared the mechanism of the anticancer effect exerted via estrogenic activities with other mechanisms.

Rubus coreanus Miquel, also known as Korean black raspberry or bokbunja, belongs to the Idaeobatus subgenus of the Rubus genus. R. coreanus is a native plant of East Asian countries, including China, Japan, and Korea. ${ }^{10}$ Bokbunja is used not only as Korean traditional beverage, but also a medicinal plant for the treatment of asthma, involuntary urination, and impotence. ${ }^{11}$ The main chemical constituents of $R$. coreanus are anthocyanins, polyphenols, triterpenoid glycosides, and tannins. ${ }^{12}$ These compounds are reported to possess anti-inflammatory ${ }^{13}$ and anti-cancer activity. ${ }^{14}$

In our study, MCF-7 human breast cancer cells were used in the E-screen assay as an in vitro model to determine the stimulatory effects of $R$. coreanus on cell proliferation. The ER $\alpha$ estrogenic activities of four compounds isolated from $R$. coreanus (3,4-dihydroxybenzoic acid, cyanidin 3-O-glucoside, nigaichigoside $\mathrm{F} 1$, and sanguiin H6) were evaluated by a molecular docking assay of the ER $\alpha$ ligand-binding domain. These 4 components were selected as a representative chemical component of anthocyanins (sanguiin H6), polyphenols (3,4-dihydroxybenzoic acid), triterpenoid glycosides (nigaichigoside F1), and tannins (cyanidin 3-O-glucoside) to find estrogenic chemical structures form various components $R$. coreanus..$^{10-14}$ The results indicated that among the tested compounds, sanguiin H6 showed the strongest effect on the estrogenic activity of $R$. coreanus through the activation of the coactivator-binding site of ER $\alpha$.

\section{Experimental}

Extraction and isolation - The dried fruits of Rubus coreanus Miquel (Korean black raspberry) were purchased from Kyoungdong Herbal Market in Seoul, in May 2016. The plant material was identified by one of the authors, Ki Hyun Kim, and a voucher specimen (BBJ-2016) was deposited at the School of Pharmacy Herbarium, Sungkyunkwan University (Suwon, Korea). The dried fruits of R. coreanus $(500 \mathrm{~g})$ were percolated three times with $80 \%$ methanol ( $2 \mathrm{~L}$, for 3 days each time) at room temperature and filtered. The filtrate was evaporated under reduced pressure to produce a crude extract $(104.5 \mathrm{~g})$. Sanguiin $\mathrm{H}-$ 6 was isolated from $R$. coreanus by using Sephadex LH20 column chromatography and preparative HPLC. Briefly, $50 \mathrm{~mL}$ of aqueous red raspberry extract was loaded onto a Sephadex LH-20 column and polyphenols such as antho- cyanins were eluted with $500 \mathrm{~mL}$ methanol:water (30:70, $\mathrm{v} / \mathrm{v})$. The fraction containing sanguiin H6 was eluted with $500 \mathrm{~mL}$ acetone:water $(70: 30, \mathrm{v} / \mathrm{v})$. The sanguiin H6-rich fraction was further purified by preparative HPLC.

Cell culture - The estrogen receptor-positive MCF-7 human breast adenocarcinoma cell line was purchased from the American Type Culture Collection (ATCC, Manassas, VA, USA). Cells were grown in RPMI 1640 medium (Catalog number: MT10041CV; Corning, Manassas, VA, USA) supplemented with 100 units $/ \mathrm{mL}$ penicillin, $100 \mu \mathrm{g} / \mathrm{mL}$ streptomycin (Catalog number: 15140122; Gibco, Carlsbad, CA, USA), and 10\% fetal bovine serum (Catalog number: FP-0500-A; Atlas Biologicals, Fort Collins, CO, USA) and incubated at $37{ }^{\circ} \mathrm{C}$ in a humidied atmosphere with $5 \% \mathrm{CO}_{2}$.

E-Screen assay - The E-screen assay for estrogenic effects on cell growth and specific protein synthesis was performed in the MCF-7 human breast adenocarcinoma cell line because of its estrogen sensitivity. The cells were incubated in 24-well culture plates at concentration of 20,000 cells/well in RPMI 1640 medium supplemented with 100 units $/ \mathrm{mL}$ penicillin, $100 \mu \mathrm{g} / \mathrm{mL}$ streptomycin, and $10 \%$ fetal bovine serum for 24 hours. The cells were treated with several concentrations of test materials (5$100 \mu \mathrm{g} / \mathrm{mL}$ for $R$. coreanus extract and $25-100 \mu \mathrm{M}$ for sanguiin H-6) in phenol red-free RPMI (Catalog number: 11835030; Gibco, Carlsbad, CA, USA) supplemented with 5\% charcoal-dextran-stripped human serum (Catalog number: IPLA-SER-CS; Innovative Research, Novi, USA) for 144 hours. For the antagonistic test, the pure estrogen receptor antagonist ICI 182780 (Catalog number: 1047; Tocris Bioscience, Bristol, UK) was added with the test materials. Estrogenic activity from serum was minimized by the use of charcoal-dextran-stripped human serum. EzCytox reagent (Catalog number: EZ-3000; Daeil Lab Service, Seoul, South Korea) was added to each well for $1 \mathrm{~h}$ and the cell viability was then calculated from the measurement of the optical density at $450 \mathrm{~nm}$ using a microplate reader (PowerWave XS; Bio-Tek Instruments, Winooski, VT, USA). ${ }^{15,16}$

Molecular docking assay - The estrogenic effects of the compounds isolated from $R$. coreanus on $\mathrm{ER} \alpha$ were evaluated by molecular docking assay of the ER $\alpha$ ligandbinding domain. Initially, the molecular model was constructed from the X-ray structure of ER $\alpha$ complexed with estradiol and a synthesized stable peptide inhibitor in the coactivator-binding groove. The atomic coordinates were obtained from the Protein Data Bank (PDB Code: $5 \mathrm{dxb}$ ) and manipulated using Discovery Studio molecular modeling package. Chain B and all water molecules of 
(A)

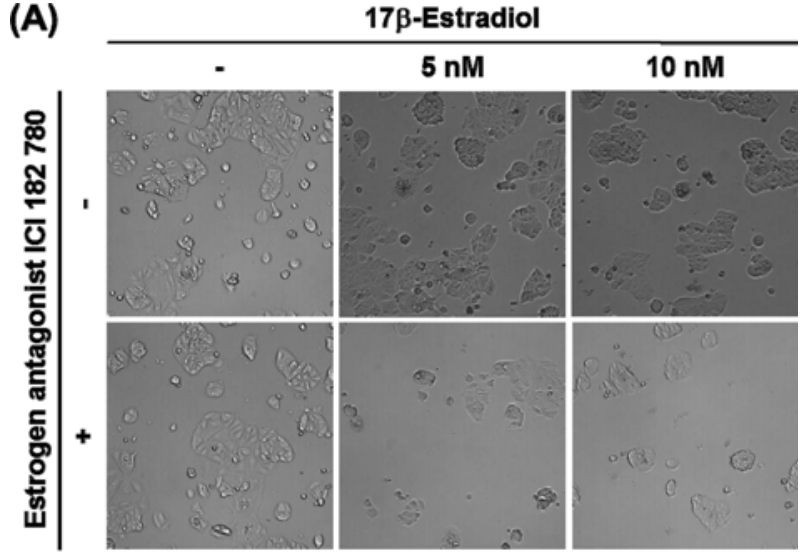

(B)

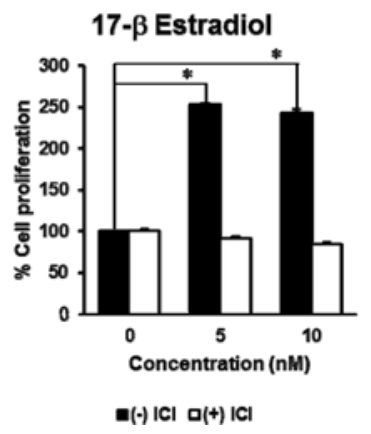

Fig. 1. The estrogenic effect of $17 \beta$-estradiol on the proliferation of MCF-7 human breast cancer cells. (A) The microscopic pictures from E-screen assay results of $17 \beta$-estradiol. (B) The comparative graph illustrates the percentage increase in cell proliferation compared with the untreated group. In this E-screen assay, 17ß-estradiol was used as a positive control. MCF-7 cells were incubated in 24-well plates and treated with tested sample in phenol red-free RPMI medium supplemented with 5\% charcoaldextran-stripped human for 144 hours. For the antagonistic test, the pure estrogen receptor antagonist ICI 182780 was added with the test materials. Ez-Cytox reagent was added to each well for 1 hour and the cell viability was then calculated from the measurement of the optical density at $450 \mathrm{~nm}$ using a microplate reader. $\mathrm{P}$ values of less than 0.001 were considered statistically significant.

chain A were deleted. To identify the docking structures of $R$. coreanus compounds that fitted into the coactivatorbinding site of $E R \alpha$, the docking simulation was implemented by CDOCKER program. All atomic charges of ligands were assigned using the Momany-Rone partial charge and CHARMM force fields. ${ }^{17-19}$

Statistical analysis - All data in our study were presented as mean \pm standard deviation (SD). Statistical significance was determined by the one-way analysis of variance (ANOVA). $\mathrm{P}$ values of less than 0.001 were considered statistically significant.

\section{Result and Discussion}

The estrogenic activity of $R$. coreanus extract was

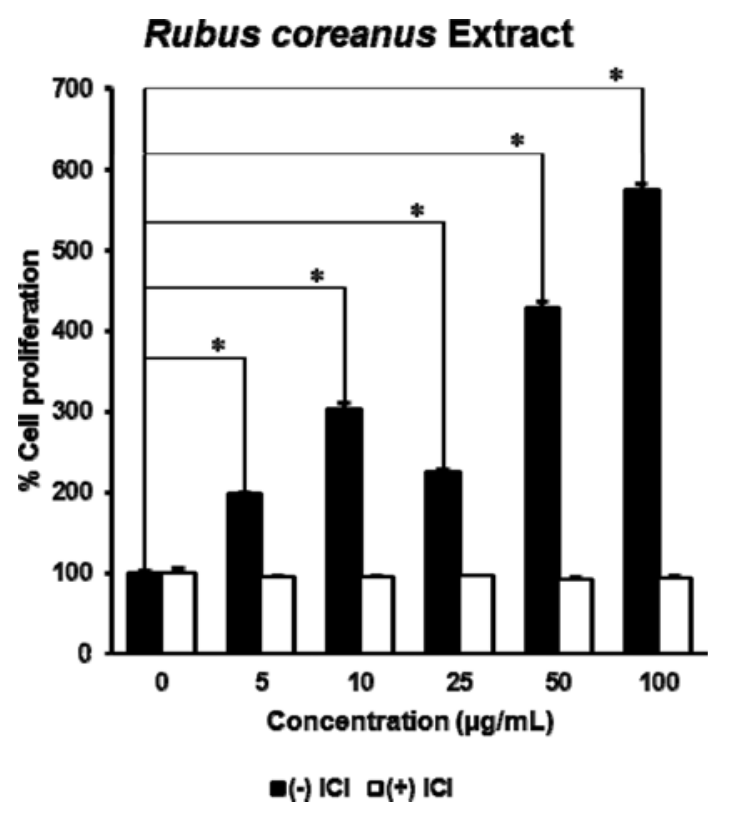

Fig. 2. The estrogenic effect of Rubus coreanus extract on the proliferation of MCF-7 cells. The extract of $R$. coreanus significantly stimulated cell growth in a dose-dependent manner, especially at the concentration of $100 \mu \mathrm{g} / \mathrm{mL}$. P values of less than 0.001 were considered statistically significant.

evaluated through the measurement of the proliferation of MCF-7 human breast adenocarcinoma cells in response to estrogen, using the E-Screen assay. In Fig. 1, the estrogenresponsive cell growth was determined by treatment with the positive control, $17 \beta$-estradiol, at 5 and $10 \mathrm{nM}$. Whereas, pre-treatment with ICI 182 780, a steroidal estrogen antagonist, completely prevented $17 \beta$-estradiolinduced the proliferation of MCF-7 cells. The extract of $R$. coreanus was found to significantly stimulate cell growth in a dose-dependent manner (Fig. 2). As shown in Fig. 2, the proliferation of MCF-7 cells increased markedly after treatment with $100 \mu \mathrm{g} / \mathrm{mL} R$. coreanus extract $(574.57 \pm 8.56 \%)$. Additionally, the proliferative effect of $R$. coreanus extract was completely prevented, in the same way as that of $17 \beta$-estradiol, by the presence of ICI 182780.

The E-screen assay showed that $R$. coreanus extract exerted estrogenic activity that induced MCF-7 breast cancer cell proliferation. Thus, the active compounds from $R$. coreanus are expected to be regulators of ER $\alpha$ transcription activity. A molecular docking assay of the $\mathrm{ER} \alpha$ ligand-binding domain was performed to analyze the estrogenic effect of $R$. coreanus compounds on ER $\alpha$. Based on a literature search, four representative isolated compounds (3,4-dihydroxybenzoic acid, cyanidin 3-Oglucoside, nigaichigoside F1, and sanguiin H6) were tested. However, the 4 compounds we selected have low 


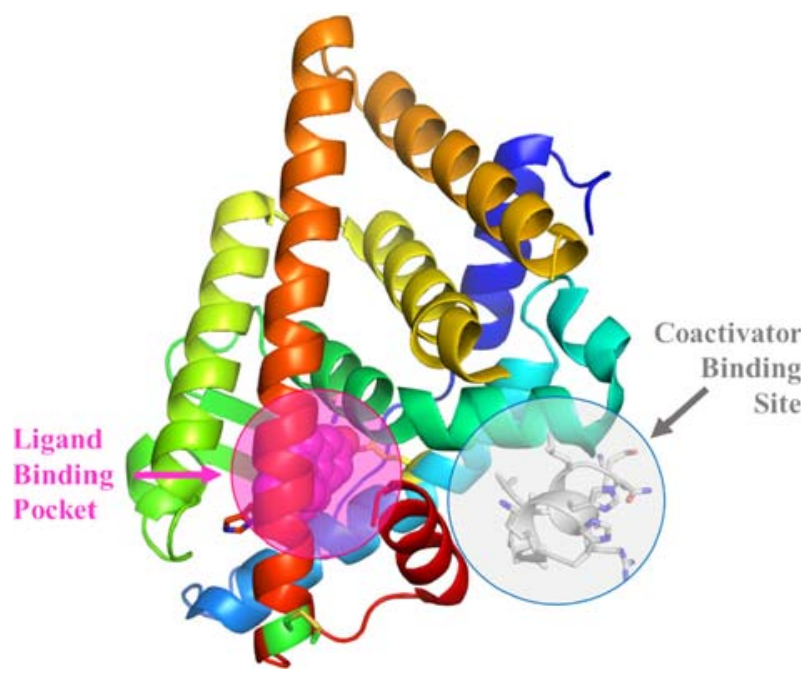

Fig. 3. Structure of ligand-binding domain of estrogen receptor $\alpha$ with the ligand-binding pocket and coactivator-binding site. Not only the binding between estrogen and its ligand binding pocket, the protein-protein interactions between coactivator proteins and their binding sites also results in cell proliferation. Competitive blockade of the coactivator binding site could effectively halt cell proliferation for breast cancer therapeutics.

binding energy or high molecular weight to ER $\alpha$. We then examined the possible action of the components on $\mathrm{ER} \alpha$ by the ER $\alpha$ coactivator. The binding energies of these compounds with the ER $\alpha$ coactivator pocket were calculated. The values for cyanidin-3-O-glucoside, 3,4dihydroxybenzoic acid, nigaichigoside F1, and sanguiin H6 were $-39.0127,-29.9156,-6.6683$, and -250.149 , respectively. The key interaction features between $R$. coreanus compounds and the coactivator pocket are the hydrophobic interactions with Val, Ile, Leu, and Met (Fig. 4), which were general protein-protein interaction related residues. Sanguiin H6 showed the strongest affinity for the ER $\alpha$ coactivator-binding site and was selected for the evaluation of the effect on the proliferation of MCF-7 human breast cancer cells.

The E-screen assay was conducted with a doseresponse study to determine the effects of $R$. coreanus compounds on MCF-7 cell proliferation (Fig. 5). The results indicated that sanguiin $\mathrm{H} 6$ and cyanidin-3-Oglucoside had an effect on the proliferation of MCF-7 cells only at $100 \mu \mathrm{M}$, the highest test concentration $(127.41 \pm 0.26 \%)$. These compounds also had the stronger affinity with the ER $\alpha$ coactivator-binding site than the other compounds. Therefore, we could not identified active ingredient that explains the effects of R. coreanus extract. Further research on other ingredients or synergies action of components is needed.

The binding between estrogen and the ligand-binding

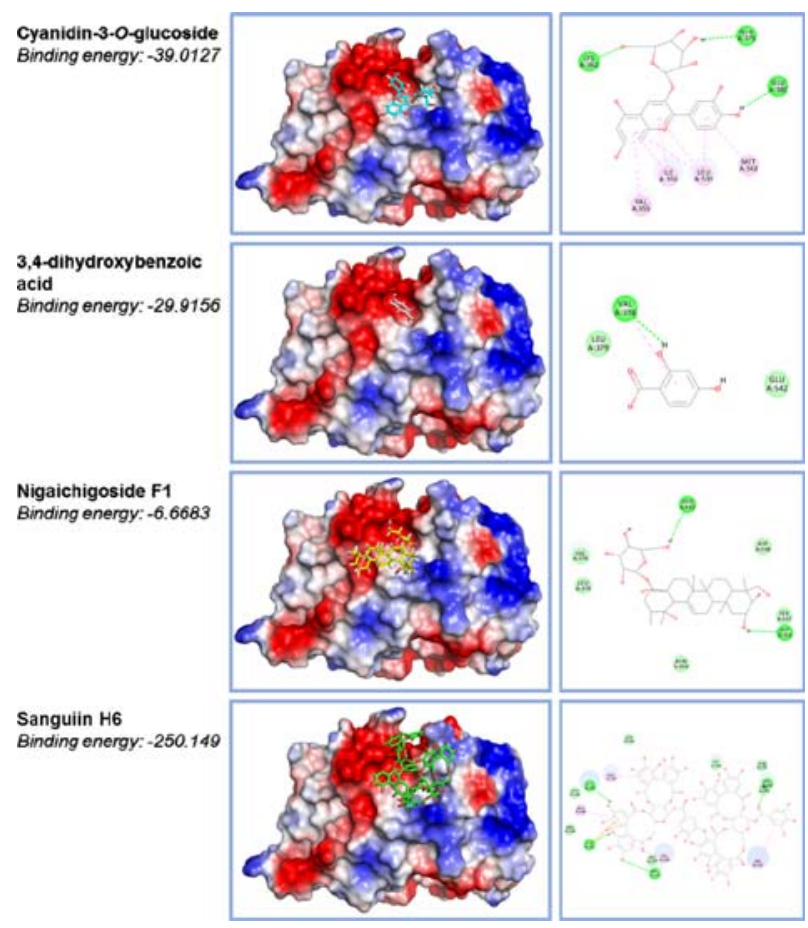

Fig. 4. The docking structures of $R$. coreanus compounds with coactivator-binding site of estrogen receptor $\alpha$. Molecular docking assay was constructed from the X-ray structure of ER $\alpha$ complexed with estradiol and a synthesized stable peptide inhibitor in the coactivator-binding groove. The atomic coordinates were obtained from the Protein Data Bank and manipulated using Discovery Studio molecular modeling package. The docking simulation was implemented by CDOCKER program. All atomic charges of ligands were assigned using the Momany-Rone partial charge and CHARMM force fields. Among four compounds isolated from $R$. coreanus, Sanguiin H6 showed the strongest affinity for the ER $\alpha$ coactivator-binding site.

domain (LBD) of the estrogen receptor (ER) initiates a biological cascade that ultimately results in cell proliferation. This binding induces a conformational change in the nuclear receptor (NR) to allow the binding of coactivator proteins, which thereby enables the further recruitment of the necessary proteins for gene transcription. ${ }^{20}$ The structure of the ER $\alpha$ ligand-binding domain with the ligand-binding pocket and coactivator-binding site is shown in Fig. 3.

Breast cancer therapeutics were initially developed with a focus on antagonists that directly block the binding of estrogen to ER-LBD. However, many recent studies have targeted the regulation of ER function by blocking the protein-protein interactions between coactivator proteins and their binding sites. ${ }^{21}$ The coactivators interact with NR-LBD through a two-turn amphipathic $\alpha$-helical motif that contains the conserved motif, LXXLL (where $\mathrm{L}$ is leucine and $\mathrm{X}$ represents any amino acid), which is known as nuclear receptor-interaction box (NR-box). ${ }^{22}$ 

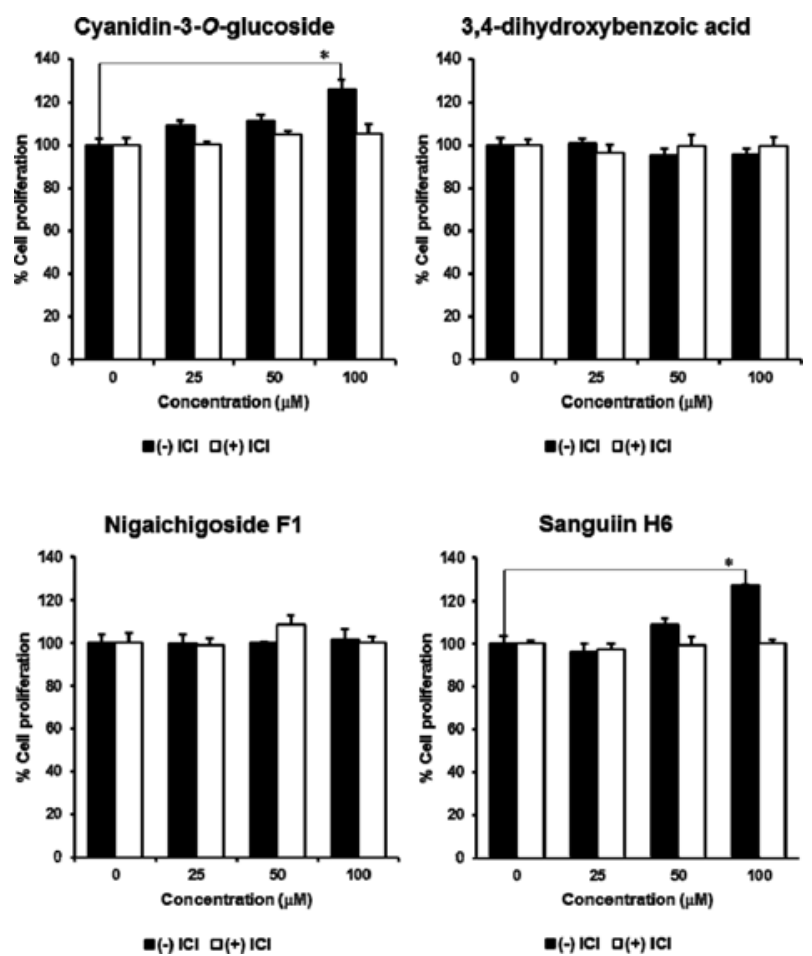

Fig. 5. The estrogenic effects of $R$. coreanus compounds on the proliferation of MCF-7 cells. Sanguiin H6 and cyanidin-3-Oglucoside slightly induced the proliferation of MCF-7 cells at the concentration of $100 \mu \mathrm{M}$. P values of less than 0.001 were considered statistically significant.

Competitive blockade of this binding site prevents recruitment of the transcription apparatus and could effectively halt cell proliferation.

E-screen assay was known as a common cell proliferation assay which allows investigating the total estrogenic activity via the increase in cell number of estrogen receptor-positive cells like MCF-7 human breast adenocarcinoma cell. ${ }^{16}$ Thus, E-screen assay was performed first to evaluate the estrogenic activity of $R$. coreanus extract. As the results, the MCF-7 cell proliferation was significantly stimulated while treated $R$. coreanus extract at the concentration of $100 \mu \mathrm{g} / \mathrm{mL}$. Many previous studies mentioned the pharmacological effects of $R$. coreanus as anti-cancer, ${ }^{23}$ anti-metastatic, ${ }^{24}$ and protective effect against oxidative stress..$^{25}$ However, to the best of our knowledge, this is one initial study about the estrogenic activity of $R$. coreanus.

Molecular docking assay was conducted to measure the binding affinity of a phytochemical from $R$. coreanus for the estrogen receptor. In this study, we focused on exploring the interaction between $R$. coreanus compounds and the coactivator binding site of estrogen receptor. Among these compounds, sanguiin H6 had the most negative interaction energy thus favorable to binding. The estrogenic activity of sanguiin $\mathrm{H} 6$ was also investigated by E-screen assay, the result showed that sanguiin H6 slightly stimulate the proliferation of MCF-7 cells at the highest test concentration of $100 \mu \mathrm{M}$.

Sanguiin H6 was considered as the potential anticancer candidate that suppressing the cell proliferation of several cancer cell lines such as A549, ${ }^{26}$ MDA-MB-231, and even MCF-7. ${ }^{23}$ There seems to be a contradiction in this present study results with the previous published data because we found that sanguiin H6 slightly stimulate the proliferation of MCF-7 cells via E-screen assay. However, it should be noted that for E-screen assay, the proliferation of human estrogen-sensitive cells was initially inhibited by human serum-borne molecules, and subsequently the estrogen-like activity of natural compounds, for example, sanguiin H6 in this case, stimulate the cell proliferation by eliminating this inhibitory effect. ${ }^{16}$ In comparison with the previous study of Park et al., the cell viability assay was conducted by adding only sanguiin $\mathrm{H} 6$ to check its cytotoxic effect on MCF-7 cells. ${ }^{23}$ In addition, many references pointed out that phytoestrogens can have both of positive and negative effects on the proliferation of breast cancer cells depend on a lot of factors such as using amount, exposure time, or the capacity of absorption and metabolism each cell lines. ${ }^{27}$ For instance, genistein is a well-known estrogen antagonist which enhanced the proliferation of MCF-7 cells by stimulating the expression of pS2 gene. ${ }^{28}$ In spite of this, genistein also worked as an anti-cancer agent while involved in the regulation of several signalling pathways like NF- $\kappa B$, Akt, proteintyrosine kinase (PTK), matrix metalloproteinases (MMPs), and $\mathrm{Bax} / \mathrm{Bcl}-2 .^{29}$

In conclusion, the E-screen assay and molecular docking analysis were conducted to evaluate the effects of the estrogenic activity of $R$. coreanus extract and its constituents on the MCF-7 human breast cancer cell line. The extract of $R$. coreanus was found to significantly stimulate cell proliferation at $100 \mu \mathrm{g} / \mathrm{mL}(574.57 \pm 8.56 \%)$. Sanguiin H6 showed the strongest affinity for the ER $\alpha$ coactivator-binding site with a binding energy of -250.149 . However, only a small effect was seen on the viability of stimulated breast cancer cells at $100 \mu \mathrm{M}(127.41 \pm 0.26 \%)$. Future studies will be conducted to confirm the molecular mechanism for the estrogenic activities of $R$. coreanus.

\section{Acknowledgements}

This research was supported by the Researcher Support Project through the Ministry of Education of the Republic 
of Korea (2017R1C1B1011984). This research was also supported by the Gachon University research fund of 2018 (GCU-2018-0319).

\section{References}

(1) Oh, C. M.; Won, Y. J.; Jung, K. W.; Kong, H. J.; Cho, H.; Lee, J. K.; Lee, D. H.; Lee, K. H. Cancer Res. Treat. 2016, 48, 436-450.

(2) Jung, K. W.; Won, Y. J.; Oh, C. M.; Kong, H. J.; Lee, D. H.; Lee, K. H. Cancer Res. Treat. 2017, 49, 306-312.

(3) Carmichael, A. R.; Mokbel, K. Arch. Plast. Surg. 2016, 43, 222-223.

(4) Irelli, A.; Cocciolone, V.; Cannita, K.; Zugaro, L.; Di Staso, M.; Lanfiuti Baldi, P. L.; Paradisi, S.; Sidoni, T.; Ricevuto, E.; Ficorella, C. Bone 2016, 87, 169-175.

(5) de Pedro, M.; Baeza, S.; Escudero, M. T.; Dierssen-Sotos, T.; Gómez-Acebo, I.; Pollán, M.; Llorca, J. Breast Cancer Res. Treat. 2015, 149, 525-536.

(6) Esteva, F. J.; Hortobagyi, G. N. Sci. Am. 2008, 298, 58-65.

(7) Jameera Begam, A.; Jubie, S.; Nanjan, M. J. Bioorg. Chem. 2017, 71, 257-274.

(8) Howell, S. J.; Johnston, S. R. D.; Howell, A. Best Pract. Res. Clin. Endocrinol. Metab. 2004, 18, 47-66.

(9) Zheng, J.; Zhou, Y.; Li, Y.; Xu, D. P.; Li, S.; Li, H. B. Nutrients. 2016, 8,495 .

(10) Lee, J.; Dossett, M.; Finn, C. E. Molecules. 2014, 19, 10524-10533.

(11) Heo, J. Donguibogam; Yeogang: Korea, 1994, pp 946-947.

(12) Li, J.; Du, L. F.; He, Y.; Yang, L.; Li, Y. Y.; Wang, Y. F.; Chai, X.; Zhu, Y.; Gao, X. M. Chem. Biodivers. 2015, 12, 1809-1847.

(13) Ju, H. K.; Cho, E. J.; Jang, M. H.; Lee, Y. Y.; Hong, S. S.; Park, J. H.; Kwon, S. W. J. Pharm. Biomed. Anal. 2009, 49, 820-827.

(14) Choung, M. G.; Lim, J. D. Korean J. Med. Crop Sci. 2012, 20, 259269.

(15) Körner, W.; Hanf, V.; Schuller, W.; Kempter, C.; Metzqer, J.;
Haqenmaier, H. Sci. Total Environ. 1999, 225, 33-48.

(16) Soto, A. M.; Sonnenschein, C.; Chung, K. L.; Fernandez, M. F.; Olea, N.; Serrano, F. O. Environ. Health Perspect. 1995, 103, 113-122.

(17) Lee, S.; Barron, M. G. PloS One 2017, 12, 1-14.

(18) Ng, H. W.; Zhang, W.; Shu, M.; Luo, H., Ge, W.; Perkins, R.; Tong, W.; Hong, H. BMC Bioinformatics. 2014, 15, 1-15.

(19) Pang, X.; Fu, W.; Wang, J.; Kang, D.; Xu, L.; Zhao, Y.; Liu, A. L.; Du, G. H. Oxid. Med. Cell. Longev. 2018, 2018, 1-11.

(20) Jordan, V. C. J. Med. Chem. 2003, 46, 883-908.

(21) McDonnell, D. P.; Chang, C. Y.; Norris, J. D. J. Steroid Biochem. Mol. Biol. 2000, 74, 327-335.

(22) Sun, A.; Moore, T. W.; Gunther, J. R.; Kim, M. S.; Rhoden, E.; Du, Y.; Fu, H.; Snyder, J. P.; Katzenellenbogen, J. A. Chem. Med. Chem. 2011, 6, 654-666.

(23) Park, E. J.; Lee, D.; Baek, S. E.; Kim, K. H.; Kang, K. S.; Jang, T. S.; Lee, H. L.; Song, J. H.; Yoo, J. E. Bioorg. Med. Chem. Lett. 2017, 27, 4389-4392.

(24) Park, E. H.; Park, J. Y.; Yoo, H. S.; Yoo, J. E.; Lee, H. L. Bioorg. Med. Chem. Lett. 2016, 26, 3291-3294.

(25) Choi, M. H.; Shim, S. M.; Kim, G. H. J. Food Sci. Technol. 2016, $53,1214-1221$

(26) Ko, H.; Jeon, H.; Lee, D.; Choi, H. K.; Kang, K. S.; Choi K. C. Bioorg. Med. Chem. Lett. 2015, 25, 5508-5513.

(27) Helferich, W. G.; Andrade, J. E.; Hoagland, M. S. Inflammopharmacology 2008, 16, 219-226.

(28) Hsieh, C. Y.; Santell, R. C.; Haslam, S. Z.; Helferich, W. G. Cancer Res. 1998, 58, 3833-3838.

(29) Lee, J. Y.; Kim, H. S.; Song, Y. S. J. Tradit. Complement. Med. 2012, 2, 96-104.
Received April 30, 2018

Revised October 1, 2018 Accepted October 3, 2018 